\title{
High sero-prevalence of hepatitis B virus and human immunodeficiency virus infections among pregnant women attending antenatal clinic at Temeke municipal health facilities, Dar es Salaam, Tanzania: a cross sectional study
}

Joel Manyahi ${ }^{1 *}$, Yohannes Msigwa $^{1}$, Francis Mhimbira ${ }^{2}$ and Mtebe Majigo ${ }^{1}$

\begin{abstract}
Background: Hepatitis B virus (HBV) and Human immunodeficiency virus (HIV) infection in pregnancy is associated with direct effect of pregnancy and potential viral transmission from mother to newborn. In Tanzania very little in known on prevalence of HBV infection and their associated factors among pregnant women in lower health facilities. The main objective of the study was to determine the prevalence of HBsAg, HIV and HBV-HIV co-infection among pregnant women attending antenatal clinics in Dar es Salaam.

Methods: This cross sectional study was conducted in three Temeke municipal health-care facilities between May 2014 and June 2014. A total of 249 pregnant women attending antenatal clinic (ANC) were consecutively enrolled in the study. A data collection tool was used to extract socio-demographic characteristics from ANC card. Commercial

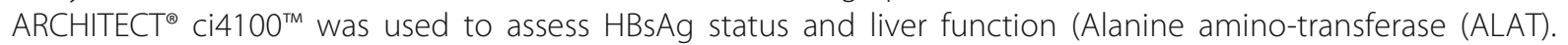
HIV status was determined by anti-HIV antibody test.

Results: Of 249 pregnant women enrolled the median age was 25 years (IQR 22-30) and most of them were married (72.4\%). The overall prevalence of HBsAg and HIV were $8.03 \%$ (95\% Cl: $5.0-12.1 \%$ ) and 17.2\% (95\% Cl: 12.8-22.5\%), respectively. HBV/HIV co-infection rate was $2.8 \%$ (95\% Cl; $1.3-5.4 \%)$. HBsAg positive rate was significantly high in women who were HIV positive $(p<0.05)$. Being employed /student were less associated with HBV infection (aOR $0.35,95 \% \mathrm{Cl} 0.13-0.95$ ). Only 3 (15\%) of pregnant women with HBsAg positive had abnormal ALAT.

Conclusions: High prevalence of HBV and HIV infections among pregnant women were reported in this setting thus calls for the national expansion of the integration of prevention of mother-to-child transmission (PMTCT) services for HBV infection.
\end{abstract}

Keywords: HBV, HBsAg, HIV, Pregnant women, Tanzania

\footnotetext{
* Correspondence: manyahijoel@yahoo.com

'Department of Microbiology and Immunology, Muhimbili University of

Health and Allied Sciences, Dar es Salaam, Tanzania

Full list of author information is available at the end of the article
} 


\section{Background}

Chronic hepatitis B virus and HIV infections remain a major global public health concern. Worldwide, about 350 million individuals are chronically HBV infected; half of them acquired infection either perinatal or in early childhood, majority found in endemic areas [1]. Annually, estimated more than 600,000 people die as a result of hepatitis $B$ virus related liver diseases [2]. The risk of progression to chronic disease is inversely proportional to the age at which infection is acquired. Infection to women during pregnancy is associated with increased risks of transmission to a newborn [3, 4]. With impaired immunity in new-born, infection acquired during this stage may lead to further progression to liver cirrhosis and hepatocellular carcinoma later in life. HBV and HIV share the common mode of transmission and co- infection in pregnancy is associated with increased morbidity, mortality and untoward outcomes to a newborn. HIV co-infection increases HBV replication, leading to higher levels of detectable virus [5], and increases likelihood of perinatal transmission of HBV.

Prevention of mother to child transmission of hepatitis $B$ virus is the most important strategy in the control of infection to newborn. Hepatitis B virus vaccine was introduced into the Tanzanian immunization and vaccine development program since2002 given at 4, 8, and 12 weeks as a DPT-HB [6]. However, the country has yet to introduce strategies to reduce the vertical transmission of HBV including screening for HBsAg, use of antivirals in the third trimester of pregnancy and at-birth prophylaxis of newborns which greatly reduces the risk of transmission [7, 8].

The prevalence of HBsAg in Tanzania varies considerably from 1.2 to $17.3 \%$ between different cohorts. Prevalence of 9.2 and $3.2 \%$ have been reported among HIV infected cohort in Southern rural and Northern Tanzania, respectively $[9,10]$. At Muhimbili national hospital, in Dar es Salaam recent studies reported HBsAg prevalence of 1.2, 3.9, 8.8 and 17.3\% among HIV children, pregnant women, blood donors and HIV adults, respectively [11-14]. These indicate that different cohort in different geographical locations at different level of health facilities have dissimilar prevalence of HBV infection. Very little in known on prevalence of Hepatitis B virus infection among pregnant women attending antenatal clinics at municipal hospital and health centers in Tanzania. Few studies reported previously were mostly conducted at referral and tertiary hospital in which population varies from district hospital and health centers.

World Health Organization (WHO) has called for integrated and standardized prevention of mother-to-child transmission (PMTCT) for HIV, syphilis and HBV [15]. Despite the call Tanzania has not scaled up PMTCT services for HBV infection. Understanding the epidemiology of Hepatitis B infections among pregnant women at different levels in Tanzania is of importance for optimizing HBV infection control strategies. Therefore, we conducted this study in Temeke municipality which has high annual growth rate in Tanzania, aiming at determining the prevalence of HBsAg and HIV among pregnant women attending antenatal clinics.

\section{Methods}

\section{Study design and settings}

This cross sectional study among pregnant women attending antenatal clinics (ANC) was conducted between May 2014 and June 2014. The study was carried out at 3 health-care facilities in Temeke municipal including Temeke hospital, Zakheem and Kizuiani health centers. Temeke Municipality is one of the three Municipalities within the Dar es Salaam City Council with a population of $1,368,881$ and an estimated growth rate of $6.6 \%$ per year compared to national annual growth rate of $2.9 \%$.

\section{Study population, sample size and sampling procedure} Participants were pregnant women attending ANC during the study period. The sample size was calculated using Kish and Lisle formula for cross sectional studies, $n=\mathrm{z}^{2} \mathrm{p}(1-\mathrm{p}) / \mathrm{d}^{2}$. Where: $\mathrm{z}=\mathrm{Z}$ score for $95 \%$ confidence interval $=1.96, \mathrm{p}=$ prevalence, $\mathrm{d}=$ tolerable error $=3 \%$. A prevalence of $3.9 \%$ was used as $\mathrm{p}$ for a previous study in Tanzania [12]. Consequently, the minimal sample size of the study population was 159 participants. However, we consecutively recruited all pregnant women who were attending ANC clinic during the study period to increase the power of our study. Therefore a total of 249 pregnant women who met eligible criteria and consenting to participate in the study were recruited. Inclusion criteria was pregnant women aged $>18$ years and women were excluded if knew their HBsAg status and if not residing in Temeke municipal.

\section{Data collection}

Data were extracted from antenatal clinic card using data standard structured questionnaires. Information on social demographic characteristics including age, education levels, occupational status and marital status were recorded.

\section{Specimen collection, transport and storage}

Five milliliters of blood were collected aseptically by veno-puncture into plain vacutainer tube. Half portion was used for HIV screening on site. Another half portion was packed into cool box then immediately delivered to central pathology laboratory at Muhimbili national hospital for further processing where sera were immediately separated after centrifugation. 


\section{Laboratory procedures}

\section{Detection of hepatitis $B$ surface antigen}

Commercially available ARCHITECT ${ }^{\ominus}$ ci4100 $^{\text {тм }}$ Integrated Immunoassay and Clinical Chemistry System (Abbott Laboratories, Abbott Park, IL, USA) was used to assess hepatitis B surface antigen (HBsAg) status. All specimens who were $\mathrm{HBsAg}$ positive, Alanine amino-transferase (ALT) were measured to assess liver function. The Architect C8000 automated analyzer was used to detect ALT levels. An Alanine amino-transferase value > $38 \mathrm{IU} / \mathrm{L}$ indicated abnormal liver function.

\section{HIV screening}

HIV infection among pregnant women was determined by anti-HIV antibody test (rapid test currently used in national algorithm for Tanzania). Alere Determine ${ }^{\mathrm{TM}}$ HIV1/2 (Alere Medical Co. Ltd. Matsudo-shi, Chibaken, Japan) was used for screening and negative results underwent no further testing. Positive samples were retested with Uni-Gold ${ }^{\mathrm{Tm}}$ HIV (Trinity Biotech Manufacturing Ltd. Bray, Ireland). Specimens that were positive on both tests were considered HIV antibody positive. All discordant specimens were sent to Muhimbili national hospital for resolution. In addition few randomly selected specimens were sent to Muhimbili national hospital for quality assurance.

\section{Data analysis}

Data were entered and analyzed using Microsoft excel 2010 and Stata version 11.2 respectively. Wilcox ranksum test and Fisher's exact test were performed to examine the difference between categorical variables. A $p$-value of $<0.05$ was considered statistically significant. We determined the association between, the outcome HBsAg and associated factors using the univariate (crude odd ratio-cOR) and multivariate logistic regression (adjusted odd ratio-aOR) were performed to identify independent factors associated with HBsAg positivity. The point estimates are presented as odd ratio (OR) and their corresponding 95\% Confidence Interval (95\% CI).

\section{Results}

\section{Study participants' characteristics}

A total of 249 pregnant women were enrolled in the study. Their age ranged from 18 to 40 years with median age of 25 years (IQR 22-30). The age group with highest proportion was $<24$ years contributing $45.8 \%$ of all participants. Nearly-half (46.2\%) of participants had secondary level of education and most participants were married $72.4 \%$. Almost forty percent (39.8\%) of the study participants were in second trimester and 52\% were petty traders (Table 1 ).
Table 1 Socio-demographic characteristics of the study participants

\begin{tabular}{|c|c|}
\hline Variables & Frequency (\%) \\
\hline Age, median (IQR) & $25(22-30)$ \\
\hline \multicolumn{2}{|l|}{ Age groups } \\
\hline$<24$ & $114(45.8)$ \\
\hline $25-30$ & $86(34.5)$ \\
\hline$>31$ & 49 (19.7) \\
\hline \multicolumn{2}{|l|}{ Education status } \\
\hline Primary & 96 (38.6) \\
\hline Secondary & $115(46.2)$ \\
\hline College & $38(15.3)$ \\
\hline \multicolumn{2}{|l|}{ Marital status } \\
\hline Single & $52(20.9)$ \\
\hline Married & $182(73.1)$ \\
\hline Cohabiting & $14(5.6)$ \\
\hline Divorced & $1(0.4)$ \\
\hline \multicolumn{2}{|l|}{ Occupation status } \\
\hline Employed & $33(13.3)$ \\
\hline Petty traders & $130(52.2)$ \\
\hline Unemployed & $64(25.7)$ \\
\hline Student & $22(8.8)$ \\
\hline \multicolumn{2}{|l|}{ HIV status } \\
\hline Positive & $43(17.3)$ \\
\hline Negative & $206(82.7)$ \\
\hline \multicolumn{2}{|l|}{ Gestation age } \\
\hline $1^{\text {st }}$ trimester & $80(32.1)$ \\
\hline $2^{\text {nd }}$ trimester & 99 (39.8) \\
\hline $3^{\text {rd }}$ trimester & $70(28.1)$ \\
\hline
\end{tabular}

\section{HBV and HIV infection}

Of 249 pregnant women, the prevalence of HBsAg was 8.03\% (95\% CI: 5.0-12.1\%). The HBsAg positive were simultaneously increasing with age, with women aged more than 35 years having the highest prevalence compared to other age groups. The HBsAg was significantly higher among women with primary school education $(12.5 \% p<0.05)$ (Table 2). Women who were married/ cohabit and those who had no employment significantly had higher prevalence of HBsAg $(p<0.05)$. The gestation age was not statistically associated with HBsAg. The overall prevalence of HIV among this cohort was $17.2 \%$ (95\% CI: 12.8-22.5). About 2.8\% (95\% CI; 1.3-5.4) of pregnant women were $\mathrm{HBV}$ and HIV co-infected. The rate of HBsAg positive was significantly higher in women who were HIV positive compared to HIV negative $(p<0.05)$. Of 20 pregnant women with HBsAg positive only $3(15 \%)$ had abnormal elevated Alanine aminotransferase (ALAT). 
Table 2 HBsAg results in relation to clinical and demographic characteristics

\begin{tabular}{llll}
\hline Variables & $\begin{array}{l}\text { HBsAg positive } \\
n=20\end{array}$ & $\begin{array}{l}\text { HBsAg negative } \\
n=229\end{array}$ & $p$-value \\
\hline $\begin{array}{l}\text { Age, median (IQR) } \\
\text { Age groups }\end{array}$ & $28(23.5-31)$ & $25(22-29)$ & $0.13^{*}$ \\
$\quad<24$ & $8(7.0)$ & $106(93.0)$ & \\
$25-30$ & $7(8.14)$ & $79(91.9)$ & 0.79 \\
$>31$ & $5(10.2)$ & $44(89.8)$ & \\
Education status & & & \\
$\quad$ Primary & $12(12.5)$ & $84(87.5)$ & 0.04 \\
$\quad$ Secondary/College & $8(5.2)$ & $145(94.8)$ & \\
Marital status & & & $0.047^{\#}$ \\
$\quad$ Single/divorced & $1(1.9)$ & $52(98.1)$ & \\
$\quad$ Married/Cohabit & $19(9.7)$ & $177(90.3)$ & \\
Occupation status & & & 0.01 \\
$\quad$ Unemployed & $10(15.6)$ & $54(84.4)$ & \\
Employed/student & $10(5.4)$ & $175(94.6)$ & \\
HIV status & & & \\
Positive & $7(16.3)$ & $193(83.7)$ & \\
Negative & $13(6.3)$ & $193.7)$ & \\
\hline
\end{tabular}

*Wilcox ranksum test $p$-value; ${ }^{*}$ Fisher's exact $p$-value

\section{Association of $\mathrm{HBsAg}$ and clinical-socio demographic characteristics}

In a univariate analysis it was found that an increase in age was associated with increased odds of $\mathrm{HBsAg}$ positive (aOR 1.05 (0.97-1.14), though this association was not statistically significant (Table 3). Pregnant women who were HIV positive were nearly three times more likely to be HBsAg positive (cOR 2.89, 95\% CI 1.08-7.73) than women who were HIV negative. Women who were married/cohabiting were also more likely to be HBsAg positive compared to those who were single/divorced (cOR 5.58, 95\% CI 0 .73-42.69). Having secondary /college education (cOR 0.39, 95\% CI 0.15-0.98) and being employed/student (cOR 0.31, 95\% CI $0.12-0.78, p<0.05)$ were significantly at reduced risk of Hepatitis $B$ virus infection. In multivariate analysis, after adjusting for potential confounding factors, Women who were employed/student remained at reduced risk of being Hepatitis B virus infected (aOR 0.35, 95\% CI 0.13-0.95).

\section{Discussion}

In the present study, the overall prevalence of HBsAg among pregnant women was $8.03 \%$. In accordance to WHO interpretation, the finding grades the study setting an area with high HBV disease endemicity [16]. Our finding is in agreement with previous studies on $\mathrm{HBsAg}$ prevalence among pregnant women from high HBV endemic areas [17-19]. In comparison to other studies conducted in Tanzania, the prevalence of HBV among pregnant women in our study was higher than that reported in rural-southern $(6.3 \%)$, northern $(4.2 \%)$ and at national hospital $(3.9 \%)[12,20,21]$. On the other hand, the prevalence was lower than that reported previously among pregnant women in Kenya (9.3\%), Uganda (11.8\%) and Cameroon (10.2\%) [22-24]. The observed variations could be attributable to geographical differences, since our study was performed in highly populated urban setting compared to previous studies in Tanzania and elsewhere.

In the current study, the prevalence of HBsAg was significantly high in pregnant women who had primary level of education compared to those with secondary/ college level of education. Our observation was in agreement to a previous conducted in Nigeria [25]. Moreover, pregnant women who had no employment and those who were married or cohabiting had high prevalence of HBsAg. These findings are in contrast with previous results reported in Uganda (Married 10.6\%), Ethiopia (low income $1.13 \%$, married $2.64 \%$ ) [23, 26]. It is not clear whether the differences observed from our study was due to high population of women who were unemployed and married/cohabiting or involvement in risky behavior in these group. Unemployment could fuel women to engage in risky sexual behavior including commercial sex, thus increasing risk of infection. Age of participants and gestation of the pregnancy were not significantly associated with high prevalence of HBsAg in line with other previous study in Ethiopia [27].

The overall prevalence of HIV among pregnant women in our study exceeded the recent reported national HIV prevalence among pregnant women [28]. Similarly, the prevalence was higher compared to results from previous studies among pregnant women in Tanzania [12, 29]. Likewise, the prevalence was higher than that reported in

Table 3 Association of positive HBsAg with clinico-demographic characteristics

\begin{tabular}{lllll}
\hline Variable & COR $(95 \% \mathrm{Cl})$ & $p$-value & aOR $(95 \% \mathrm{Cl})$ & $p$-value \\
\hline Age in years & $1.05(0.97-1.14)$ & 0.24 & $1.02(0.93-1.13)$ & 0.624 \\
HIV & $2.89(1.08-7.73)$ & 0.035 & $2.24(0.77-6.48)$ & 0.137 \\
Married/Cohabiting & $5.58(0.73-42.69)$ & 0.098 & $0.66(0.53-41.0)$ & 0.165 \\
Secondary/College education & $0.39(0.15-0.98)$ & 0.046 & $0.41(0.15-1.1)$ & 0.076 \\
Employed/Student & $0.31(0.12-0.78)$ & 0.013 & $0.35(0.13-0.95)$ & 0.039 \\
\hline
\end{tabular}

COR crude odd ratio, $\mathrm{Cl}$ confidence interval, aOR adjustable odd ratio 
recent studies in Uganda (9.3\%) and Ethiopia (6.6\%), which are HIV endemic areas [23, 27]. The observed differences to this study maybe attributable to risk of sexual behavior since most of participants in the current study were less than 24 years, hence likely to be involved in high risky sexual behavior like prostitution. Likewise, this is the age were most participants may commence unprotected sexual interaction. In addition the study site has estimated population growth rate of $6.6 \%$ per year compared to national annual population growth rate of $2.9 \%$, displaying high sexual activities in this setting [30], explaining the observed finding.

Prevalence of HBsAg was significantly high in pregnant women who were HIV positive compared to those who were HIV negative $(p<0.05)$. This finding is in contrast to HBsAg prevalence reported among HIV-positive and HIV-negative pregnant women at a tertiary hospital in Tanzania [12]. On the Contrary, studies from SubSaharan Africa have reported similar positivity rate of HBsAg prevalence among HIV-positive and HIV negative pregnant women [23, 31, 32]. The predominant mode of transmission is still thought to be childhood/ perinatal in this country. The higher prevalence of HBV infection among HIV patients observed in our study could be due to those patients being less able to clear it.

The rate of HBV and HIV co-infection in our study was $2.8 \%$. This rate was higher than that reported previously from a national hospital in Dar es Salaam, Tanzania [12]. Furthermore, the prevalence of HBV/HIV co-infection among pregnant women found in our study was higher than the previous reports in Nigeria $(0.24 \%)$, Ethiopia (0.6\%) and Cameroon (1.5\%) [24, 33, 34]. However, the prevalence of HBsAg in the current study was lower than that reported from a recent study among pregnant women in South Africa (3.1\%) [35]. The observed low HBV/HIV co-infection rates in settings of high HBV endemicity could be explained by the fact that the main routes of transmission of HBV in SubSaharan Africa could be vertical and likely occurs in early childhood.

Our study found that employment and being students were independent factors associated with reduced risk of $\mathrm{HBV}$ infection $(\mathrm{aOR}=0.35 ; 95 \% \mathrm{CI} 0.13-0.95)$. The finding of employment and students being protective to HBV infections was contrary to studies conducted earlier in Tanzania and elsewhere within the region [12, 27, 32]. Similar to other previous reports, marital status and education levels were not independently associated with HBV infections. HIV was also not independently associated with HBV infection, despite showing association in bivariate analysis. Similar finding was observed in previous studies among pregnant women in Malawi, Ethiopia and Cameroon [27, 32, 36]. In spite of lack of association between HBV and HIV in our study, prevalence of these infections remains high in Tanzania, as observed in the current study.

Our study had some limitations; detection of other HBV surrogate markers (HBeAg, HBc-antibodies, HBV DNA) and Hepatitis $C$ virus were not possible due to financial constraints. We did not ascertain risk factors for HBV infection because of recall biases and design of the study. Despite that, our findings are based on a substantial sample size and the inclusion of municipal and health centers minimized the selection biases, since these are catchment settings where both uncomplicated and complicated cases are first seen.

\section{Conclusions}

Our study findings suggest high prevalence of HBV and HIV infection among pregnant women in municipal health facilities in Dar es Salaam, Tanzania, while HBV/HIV coinfection is low. The results suggest that perinatal transmission is a common mode of HBV transmission in our setting. Based on our finding, we advocate integrating HBV infection into PMTCT program in Tanzania. Testing for HBsAg should be recommended for every pregnant women and possible prevention of mother-to-child transmission measures should be considered.

\section{Abbreviations \\ ALAT: Alanine aminotransferase; ANC: Antenatal clinic; aOR: Adjustable odd ratio; Cl: Confidence interval; COR: Crude odd ratio; DPT-HB: Diptheria, \\ Pertusis, tetanus and Hepatitis B vaccine; HBCAg: Hepatitis B core antigen; HBeAg: Hepatitis B envelope antigen; HBsAg: Hepatitis B surface antigen; HBV: Hepatitis B virus; HIV: Human immunodeficiency virus; IQR: Interquartile range; PMTCT: Prevention of mother to child transmission; WHO: World Health Organization}

\section{Acknowledgments}

We are grateful to central pathology laboratory of Muhumbili national hospital for their support in testing our specimens.

Funding

The study was funded by Tanzania High Education Loans Board.

Availability of data and materials

The datasets analyzed for this study can be obtainable from the corresponding author on special request.

\section{Authors' contributions}

JM conceived the research idea and drafted the manuscript. YM participated in data collection. FM performed statistical analysis and revised the manuscript. MM participated in design of the study and critically revised the manuscript. All authors read and approved the final manuscript.

\section{Competing interests}

The authors declare that they have no competing interests.

\section{Consent for publication}

Not applicable.

\section{Ethics approval and consent to participate}

Ethical clearance to perform the study was obtained from the Muhimbili University of Health and Allied Sciences Senate Research and Publication Committee. Permission to perform the study was granted by Temeke municipal administration. Written informed consent was obtained from pregnant women before enrollment. The results of laboratory tests performed in this study were shared to the respective ANC clinics for further management. 


\section{Publisher's Note}

Springer Nature remains neutral with regard to jurisdictional claims in published maps and institutional affiliations.

\section{Author details}

'Department of Microbiology and Immunology, Muhimbili University of Health and Allied Sciences, Dar es Salaam, Tanzania. ${ }^{2}$ Ifakara Health Institute, Dar es Salaam, Tanzania.

Received: 6 August 2015 Accepted: 29 March 2017

Published online: 07 April 2017

\section{References}

1. Lavanchy D. Hepatitis B, virus epidemiology, disease burden, treatment, and current and emerging prevention and control measures. J Viral Hepat. 2004; 11:97-107.

2. Goldstein ST, Zhou F, Hadler SC, Bell BP, Mast EE, Margolis HS. A mathematical model to estimate global hepatitis B disease burden and vaccination impact. Int J Epidemiol. 2005:34:1329-39.

3. Edmunds WJ, Medley GF, Nokes DJ, Hall AJ, Whittle HC. The influence of age on the development of the hepatitis B carrier state. Proc Biol Sci. 1993; 253:197-201.

4. Hyams KC. Risks of chronicity following acute hepatitis B virus infection: a review. Clin Infect Dis. 1995;20:992-1000.

5. Thio CL. Hepatitis $B$, and human immunodeficiency virus coinfection. Hepatology. 2009:49(5 Suppl):S138-45.

6. Ministry of Health and Social Welfare Tanzania. Expanded programme on immunization, comprehensive multiyear plan. 2nd ed. Dar es Salaam: Government Press; 2007. p. 5-7.

7. Giles ML, Grace R, Tai A, Michalak K, Walker SP. Prevention of mother-to-child transmission of hepatitis B virus (HBV) during pregnancy and the puerperium: current standards of care. Aust N Z J Obstet Gynaecol. 2013;53:231-5.

8. Gentile I, Zappulo E, Buonomo AR, Borgia G. Prevention of mother-to-child transmission of hepatitis B virus and hepatitis C virus. Expert Rev Anti Infect Ther. 2014;12(7):775-82.

9. Franzeck FC, Ngwale R, Msongole B, Hamisi M, Abdul O, Henning L, et al. Viral hepatitis and rapid diagnostic test based screening for HBsAg in HIVinfected patients in rural Tanzania. PLoS One. 2013;8:e58468.

10. Debes JD, Stauffer WM, Jacobson M, Boulware DR. Low rates of hepatitis B and human immunodeficiency virus coinfection in rural northern Tanzania. J Glob Infect Dis. 2015;7:47-8.

11. Telatela SP, Matee MI, Munubhi EK. Seroprevalence of hepatitis B and C viral co-infections among children infected with human immunodeficiency virus attending the paediatric HIV care and treatment center at Muhimbil National Hospital in Dar-es-Salaam, Tanzania. BMC Public Health. 2007;7:338.

12. Rashid S, Kilewo C, Aboud S. Seroprevalence of hepatitis B virus infection among antenatal clinic attendees at a tertiary hospital in Dar es Salaam, Tanzania. Tanzan J Health Res. 2014:16:1.

13. Matee MI, Magesa PM, Lyamuya EF. Seroprevalence of human immunodeficiency virus, hepatitis B and C viruses and syphilis infections among blood donors at the Muhimbili National Hospital in Dar es Salaam, Tanzania. BMC Public Health. 2006:6:21.

14. Nagu TJ, Bakari M, Matee M. Hepatitis A, B and C viral co-infections among HIV-infected adults presenting for care and treatment at Muhimbili National Hospital in Dar es Salaam, Tanzania. BMC Public Health. 2008;8:416.

15. Wang AL, Qiao YP, Wang LH, Fang LW, Wang F, Jin X, et al. Integrated prevention of mother-to-child transmission for human immunodeficiency virus, syphilis and hepatitis B virus in China. Bull World Health Organ. 2015; 93:52-6.

16. Hou J, Liu Z, Gu F. Epidemiology and prevention of hepatitis B virus infection. Int J Med Sci. 2005:2:50-7.

17. MacLean B, Hess RF, Bonvillain E, Kamate J, Dao D, Cosimano A, et al. Seroprevalence of hepatitis B surface antigen among pregnant women attending the Hospital for Women \& Children in Koutiala, Mali. S Afr Med J. 2011;102:47-9

18. Luka SA, Ibrahim MB, lliya SN. Sero-prevalence of hepatitis B surface antigen among pregnant women attending Ahmadu Bello University Teaching Hospital, Zaria, Nigeria. Niger J Parasitol. 2008;29:38-41.

19. Olokoba AB, Salawu FK, Danburam A, Olokoba LB, Midala JK, Badung LH, et al. Hepatitis B virus infection amongst pregnant women in North-eastern Nigeria- a call for action. Niger J Clin Pract. 2011;14:10-3.
20. Menendez C, Sanchez-Tapias JM, Kahigwa E, Mshinda H, Costa J, Vidal J, et al. Prevalence and mother-to-infant transmission of hepatitis viruses $B, C$, and E in Southern Tanzania. J Med Virol. 1999;58:215-20.

21. Msuya SE, Mbizvo EM, Hussain A, Sam NE, Stray-Pedersen B. Seroprevalence of hepatitis $B$ and $C$ viruses among women of childbearing age in Moshi Urban, Tanzania. East Afr Med J. 2006:83:91-4.

22. Okoth F, Mbuthia J, Gatheru Z, Murila F, Kanyingi F, Mugo F, et al. Seroprevalence of hepatitis B markers in pregnant women in Kenya. East Afr Med J. 2006;83:485-93.

23. Bayo P, Ochola E, Oleo C, Mwaka AD. High prevalence of hepatitis B virus infection among pregnant women attending antenatal care: a cross-sectional study in two hospitals in northern Uganda. BMJ Open. 2014;4:e005889.

24. Noubiap JJ, Nansseu JR, Ndoula ST, Bigna JJ, Jingi AM, Fokom-Domgue J. Prevalence, infectivity and correlates of hepatitis B virus infection among pregnant women in a rural district of the Far North Region of Cameroon. BMC Public Health. 2015;15:454

25. Anaedobe CG, Fowotade A, Omoruyi CE, Bakare RA. Prevalence, sociodemographic features and risk factors of Hepatitis B virus infection among pregnant women in Southwestern Nigeria. Pan Afr Med J. 2015;20:406. doi: 10.11604/pamj.2015.20.406.6206.

26. Tegegne D, Desta K, Tegbaru B, Tilahun T. Seroprevalence and transmission of Hepatitis B virus among delivering women and their new born in selected health facilities, Addis Ababa, Ethiopia: a cross sectional study. BMC Res Notes. 2014;7:239.

27. Zenebe $Y$, Mulu W, Yimer M, Abera B. Sero-prevalence and risk factors of hepatitis $B$ virus and human immunodeficiency virus infection among pregnant women in Bahir Dar city, Northwest Ethiopia: a cross sectional study. BMC Infect Dis. 2014;14:118.

28. Manyahi J, Jullu B, Abuya MI, Juma J, Ndayongeje J, Kilama B, Sambu V, Nondi J, Rabiel B, Somi G, Matee MI. Prevalence of HIV and syphilis infections among pregnant women attending antenatal clinics in Tanzania, 2011. BMC Public Health. 2015;15:501.

29. Swai RO, Somi G, Matee MI, Killewo J, Lyamuya EF, Kwesigabo G, Tulli T, Kabalimu TK, Ng'ang'a L, Isingo R, Ndayongeje J. Surveillance of HIV and syphilis infections among antenatal clinic attendees in Tanzania-2003/2004. BMC Public Health. 2006;6:91

30. United Republic of Tanzania. National Bureau of Statistics Ministry of Finance Dar es Salaam and Office of Chief Government Statistician President's Office, Finance, Economy and Development Planning Zanzibar. Population Distribution by Age and Sex. 2013

31. Rouet F, Chaix ML, Inwoley A, Msellati P, Viho I, Combe P, et al. HBV and HCV prevalence and viraemia in HIV-positive and HIV-negative pregnant women in Abidjan, Cote d'Ivoire: the ANRS 1236 study. J Med Virol. 2004;74:34-40.

32. Fomulu NJ, Morfaw FL, Torimiro JN, Nana P, Koh MV, William T. Prevalence, correlates and pattern of Hepatitis B among antenatal clinic attenders in Yaounde-Cameroon: is perinatal transmission of HBV neglected in Cameroon? BMC Pregnancy Childbirth. 2013;13:158.

33. Ikeako L, Ezegwui H, Ajah L, Dim C, Okeke T. Seroprevalence of human immunodeficiency virus, hepatitis B, hepatitis C, syphilis, and co-infections among antenatal women in a tertiary institution in South East, Nigeria. Ann Med Health Sci Res. 2014;4(6):954-8.

34. Ramos JM, Toro C, Reyes F, Amor A, Gutierrez F. Seroprevalence of HIV-1, HBV, HTLV-1 and Treponema pallidum among pregnant women in a rural hospital in Southern Ethiopia. J Clin Virol. 2011;51:83-5.

35. Thumbiran NV, Moodley D, Parboosing R, Moodley P. Hepatitis B and HIV co-infection in pregnant women: indication for routine antenatal hepatitis $B$ virus screening in a high HIV prevalence setting. S Afr Med J. 2014;104:307-9.

36. Ahmed SD, Cuevas LE, Brabin BJ, Kazembe P, Broadhead R, Verhoeff FH, et al. Seroprevalence of hepatitis B and C and HIV in Malawian pregnant women. J Infect. 1998;37:248-51. 